\title{
Molecular Methods Developed for the Identification and Characterization of Candida Species
}

\author{
Danielly Beraldo dos Santos Silva, Kelly Mari Pires de Oliveira, Alexéia Barufatti Grisolia* \\ Universidade Federal da Grande Dourados, Dourados, MS, Brazil
}

Received: 21 June, 2016; Accepted: 01 September, 2016; Published: 10 September, 2016

*Corresponding author: Grisolia AB, Faculty of Biological and Environmental Sciences (FCBA), Federal University of Grande Dourados (UFGD). Rodovia Dourados-Itahum KM 12, P.O. Box:533, Zip Code:79804-970, Dourados/ Mato Grosso do Sul/ Brazil, Tel: +55 (067) 34102223; E-mail: alexeiagrisolia@ufgd.edu.br

\begin{abstract}
Candida species are commensally microorganisms in healthy individuals, but is also the most opportunistic fungal pathogen of human. From benign skin-mucosal forms to invasive ones, Candida infection, can compromise various organs systematic and cause diseases in immunocompromised or critically ill patients. Hence the accurate identification and characterization of the disease-causing strains is crucial for diagnosis, clinical treatment and epidemiological studies of candidacies. Molecular methods the identification and characterization support gives in epidemiological research and in development of novel antifungal with specific molecular targets, especially in the case of resistant Candida species. This review aims to describe the main methods used in the identification and characterization the Candida species and discusses future perspectives.
\end{abstract}

Keywords: Candidiasis; ITS; Genomes; Molecular typing

\section{Introduction}

Candida species commensally inhabit the human body. The relationship between an autochthonous Candida species and its human host can be affected by pathological, physiological, mechanical and iatrogenic factors. In this sense, Candida species can cause several types of infections with a wide spectrum of clinical presentations, from superficial benign forms to invasive ones that compromise several organs, leading to host death [1]. Candida species exhibit a large clinical relevance, therefore is necessary to correctly identify and characterize the isolates at the molecular level for understanding the spread of species and the mechanisms of their resistance to antifungal agents [2].

The resistance of Candida species to antifungal agents can often be attributed to a mutation gene or other alteration (such as increased expression) of the drug target [3]. In this sense, were developed several effective molecular methods for the identification and characterization of Candida species. These methods support gives in epidemiological research and in development of novel antifungal with specific molecular targets, especially in the case of resistant Candida species.
This review summarizes the insights the main methods used in the identification and characterization the Candida species and discusses future perspectives: omics technologies for characterization of Candida species.

\section{General features: Candida species}

Candida species are taxonomically classified in the kingdom Fungi, phylum Ascomycota, class Saccharomycetes, family Saccharomycetaceae, and genus Candida. These yeasts are unicellular microorganisms that are pleomorphic and ovoid or spherical in shape with an incomplete sexual cycle. Candida species commensally inhabit the human body and can be found in the respiratory tract, gastrointestinal tract, vaginal mucosa, oral cavity, and skin of healthy individuals [4]; they can also be isolated from plants, water, soil, and other environments. Moreover, they can degrade proteins and carbohydrates as a source of carbon and nitrogen, which are essential for their development [5].

The relationship between an autochthonous Candida species and its human host can be affected by pathological, physiological, mechanical, and iatrogenic factors. In this sense, Candida species can cause several types of infections with a wide spectrum of clinical presentations, from superficial benign forms to invasive ones that compromise several organs, leading to host death [1].

There are close to 200 different Candida species, five of which Candida albicans, Candida tropicalis, Candida glabrata, Candida krusei, and Candida parapsilosis [subdivided into C. parapsilosis, Candida orthopsilosis, and Candida metapsilosis] (Figure 1) are involved in more than $90 \%$ of invasive infections [6,7]. Other emergent Candida species, such as Candida guilliermondii, Candida dubliniensis, Candida lusitaniae, Candida kefyr, Candida rugosa, Candida famata, Candida utilis, Candida lipolytica, Candida norvegensis, and Candida inconspícua, also have clinical relevance, and have been identified as causative agents of superficial and systemic itches $[7,8]$.

C. albicans is considered the most frequently isolated species from patients with superficial and invasive infections of different 


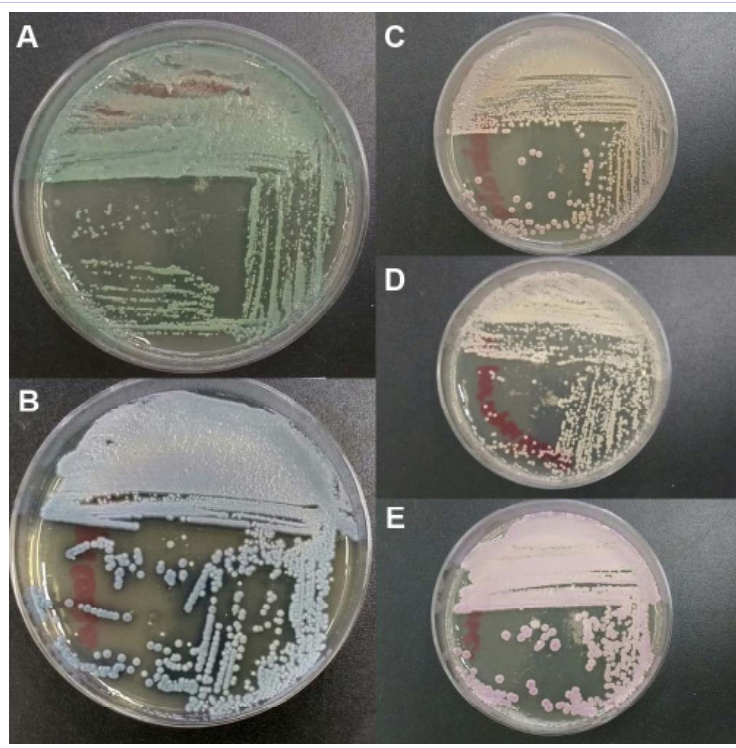

Figure 1: Candida species clinical, isolated on selective and differential CHROMagar $^{\mathrm{TM}}$ Candida DIFICO ${ }^{\circledR}$. A) Candidaalbicans; B) Candidatropicalis; C) Candidaglabrata; D) Candidaparapsilosis; E) Candidakrusei..

anatomical sites in case studies from around the world [9]. Its main pathogenic mechanisms and virulence factors appear to be its ability to adhere to different mucous membranes and epithelia, its ability to produce filamentous structures that assist in tissue invasion, and its production of enzymes such as proteinases and phospholipases. Such species are naturally sensitive to antifungal agents when used systemically, but cases of acquired resistance to azolics, in particular to fluconazole, have been described in patients that receive long-term antifungal therapy [10].

In Latin American countries, especially Brazil, C. tropicalis is detected in $20-24 \%$ of blood-borne infections $[10,11]$, mainly in elderly patients and those with conditions such as neutropenia and Diabetes mellitus [12]. Clinical isolates of this species are typically sensitive to amphotericin B and triazoles [13]; however, cases of resistance to these drugs, especially to fluconazole, have been recently reported and described [14].

The species C. glabrata and, C. krusei also have pathogenic potential $[10,11]$. C. glabrata ranks second among isolates from blood-borne infections in the United States. This species has also experienced an increase in the percentage of fluconazoleresistant isolates as well the incidence of isolates with reduced sensitivity to amphotericin B and cross-resistance to other drugs from the azole class [10]. C. krusei has been shown to be an occasional hospital pathogen, particularly in patients with hematological malignancies and/or that undergoing bone marrow transplantation. C. krusei is naturally resistant to fluconazole [15].

C. parapsilosis has been isolated from health professional studies and parenteral nutrition solutions [16]. This yeast has been recognized as a major cause of candidemia related to infections that begin from the skin [17]. In general, clinical isolates of this species are sensitive to most antifungal agents, especially amphotericin B and azoles. However, in clinical studies, isolates with reduced sensitivity to fluconazole have been reported [18]. Together, Candida species exhibit a large clinical relevance due to their incidence of colonization and infection of the human body. It is therefore necessary to correctly identify and diagnose the yeast species responsible for an infection and subsequently prescribe the proper antifungal agent for treatment.

Phenotypic methods used in the identification and characterization of Candida species are based on analysis of morphological and biochemical profile of these organisms. The most widely used phenotypic methods for identification and characterization the Candida species are observation of microscopic structures, evaluation tests of enzyme activity and assimilative capacity and substrate fermentation [19].

Several commercial products and systems have been developed the aim of solve some difficulties experienced by the clinical microbiology laboratories in the diagnosis of infections caused by Candida species [20], for example Agar containing chromo gens, kits and panels semi or full automated to the presumed or definitive identification of the most prevalent species $[21,22]$. The use of only phenotypic testing is not highly effective for identification of Candida species because some species has few morphological and biochemical variations, therefore phenotypic and molecular methods can be used in together to increase reliability in the identification of these species.

\section{Identification and Molecular Characterization}

In recent decades, molecular biological techniques have been used to better understand the pathogenicity of Candida species and to expand the search for new molecular drug targets. Candida species are diploid, heterozygous, and contain a plastid genome, features which recent genomic approaches have been able to better characterize. In 1996, the Candida Genome Sequencing Project was initiated with a goal of sequencing the genome of C. albicans SC5314 $[23,24]$. This goal necessitated the use of bioinformatics tools that were able to predict and annotate genes in the sequence. The result was the description of 6,354 genes, although the DNA sequences of some specific chromosomes had still not been determined.

Comparison of the $C$. albicans genome with the genomes of other fungal species allowed for the identification of several specific genes that could be potential targets for antifungal therapies. It was observed that compared with other fungi, the coding sequence of the C. albicans genome was rich in short tandem repeats (STRs). It was also possible to identify and conduct detailed analyses of multi genic families found in $C$. albicans, many of which were related to its pathogenicity [23].

In 2007, Van Het Hoog, et al. [25] provided the complete sequence of the $C$. albicans SC5314 genome (15.845 Mb organized into 8 chromosomes). In addition to its utility for genetic mapping, it provided updates for certain genome characteristics, including the discovery of an additional transcription factor gene family, information concerning the chromosomal locations of gene families, and a review of the open reading frame (ORF) list previously annotated by Braun, et al. [23]. 


\section{Molecular Methods Developed for the Identification and Characterization of Candida Species}

To determine the genetic characteristics underlying the biological and pathogenic diversity of the different Candida species, Butler, et al. [26] proposed sequencing of $C$. albicans (W0-1), C. tropicalis, C. parapsilosis, C. guilliermondii, C. lusitaniae, and Lodderomyceselongisporus (a close relative of $C$. parapsilosis) along with subsequent comparisons to the already sequenced genomes of C. albicansSC5314 and Debaryomyceshansenii, a marine yeast rarely associated with disease [23-25,27].

The genomes of the species sequenced by Butler, et al. [26] ranged in both size (between 10.6 to $15.5 \mathrm{Mb}$ ) and composition of protein-coding genes (5,733-6,318 genes) across the different species. The authors also identified 64 gene families that appeared to be related to Candida pathogenicity. Six of these families had been previously shown to be associated with pathogenicity, including the ERG3 gene, which is involved in the ergosterol biosynthesis pathway. The dissemination of $C$. albicans and Non-albicans Candida (NAC) genomes in the public domain accelerated research on the biological and molecular mechanisms associated with the pathogenicity of these species.

Due to the fact that some species of Candida present few easily identifiable morphological and biochemical variations, molecular biological techniques have been used to overcome the limitations of phenotypic identification methods. Fungal molecular systematic is based primarily on the analysis of mitochondrial genes (mtDNA) and ribosomal DNA (rDNA) [28].

The mitochondrial cytochrome $\mathrm{C}$ oxidase subunit 1 (COI) was proposed for molecular identification at the species level [29]. This gene was adopted by the Consortium for the Barcode of Life for the classification of all organismal groups, including fungi [30]. COI works reasonably well as a barcode in some fungi genera, such as Penicillium [31]; however, results in other groups that have been experimentally examined are inconsistent, limiting this gene's use [32].

Ribosomal genes are conserved among all known organisms. As a consequence, this gene allows for the joint reconstruction of both prokaryotic and eukaryotic phylogenies. On the other hand, the nucleotide substitution rate is low in the 18S rRNA gene [33] often preventing discrimination between closely related species. Thus, species that exhibit differences at the ribosomal gene level have likely already been diverging for at least a few million years.

For discrimination of closely related filamentous fungal species and yeasts, the Internal Transcribed Spacer regions (ITS 1 and ITS 2), located between the 18S, 5.8S, and 28S rRNA genes, and the D1/D2 region, located in the larger rDNA subunit, have shown to be successful in species-level identification (Figure 2). In both ITS1 and ITS2, there are 100-200 tandem repeats, which contain both highly conserved domains and variable domains, respectively [34]. These regions have been adopted for use in bar-coding for most fungi genera and are considered standard markers by the Consortium for the Barcode of Life [35].

Kurztzman, et al. [36] described the presence of extensive genetic differentiation in the D1/D2 region, allowing for the differentiation of ascomycetes. Since then, this region, together

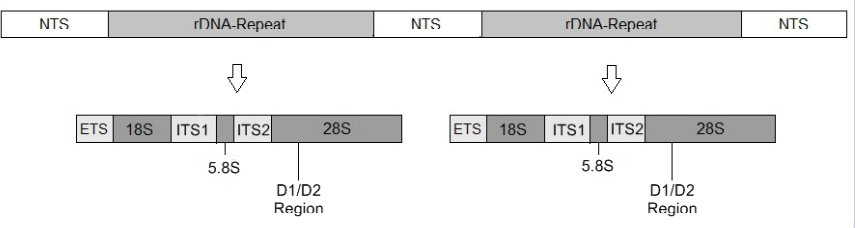

Figure 2: Structure of fungal DNA. NTS: Non- transcribed spacer, ETS: External transcribed spacer, ITS: Internal transcribed spacer, genes $18 \mathrm{~S}, 5.8 \mathrm{~S}$ and $28 \mathrm{~S}$ ribosomal the DNA.

with the ITS regions, have been widely used for both identification and establishment of evolutionary relationships (phylogenies) of several fungi species, including Candida species. Phylogenies inferred from molecular data have been used to clarify the main evolutionary divisions between taxa, as well as helping in the identification process of higher taxonomic groups, including the Fungi kingdom [28].

Evolutionary histories as elucidated by phylogenetic analysis are normally illustrated as branching, treelike diagrams that represent estimates of inherited relationships between molecules (phylogenetic tree), organisms, or both [37]. There are three classes of phylogenetic methods: distance-based, character-based, and Bayesian inference-based. In models that use distances for phylogenetic reconstruction, two steps are necessary: distance calculation and construction of the topology.

For distance-based calculation, calculated matrices are used for pair wise comparisons between the aligned sequences based on a replacement model, i.e., an evolutionary model of these sequences. Among the most widely used models for phylogenetic analysis in Candida are the P Distance, Juckes-Cantor, Kimura 2-parameter, Tajima and Nei, Tamura 3-Parameter, Tamura and Nei, Gamma-Poisson, and PAM Distance models. The most common algorithms used to order the calculated distances between the macromolecular sequences contained in the matrix into a topology are UPGMA (Unweighted Pair Group Method with Arithmetic Mean), Neighbor-Joining, and Minimum Evolution [38].

The character-based models are directly inspired by the cladistics methods of Maximum Parsimony and Maximum Likelihood [39]. For models based on Bayesian inference, the parameters are considered random variables in which the uncertainty about values is measured by the distribution of posterior probabilities [40].

Many techniques are used for molecular characterization of Candida species, such as Restriction Fragment Length Polymorphism (RFLP) [41], DNA fingerprinting [42], electrophoretic karyotyping [43], Random Amplified Polymorphic DNA (RAPD) [44], Multilocus Enzyme Electrophoresis (MLEE) [45], and sequencing of microsatellites [46]. However, in order to estimate genetic distances and infer phylogenetic relationships between species that can be easily evaluated in terms of probability models, the most suitable techniques involve gene sequencing and produce topologies built from nucleotide or amino acid sequences. 
One of the techniques used for molecular characterization are the microarrays. Microarray-based systems offer an attractive outlook for the of strain typing. They can offer high level of specificity, sensitivity and through put capacity. For typing molecular, microarrays can be used to identification and get the different sequence variants of specific genes or regions and ITSs, in particular. Ongoing sequencing projects in pathogenic yeasts also enable quite straightforward designing of whole-genome DNA microarrays [47].

Multilocus Sequence Typing (MLST), a sequencing-based method, was initially developed for both clone identification and bacterial pathogen typing [48]. This method analyzes nucleotide polymorphisms in essential genes fragments, the "housekeeping genes," and can produce sequences of up to $500 \mathrm{bp}$ [48-50], generating a molecular characterization with high discriminatory power and reproducibility.

The MLST technique has been used previously for several Candida species, among them, C. albicans [49,51]. Based on collaborative work, a set of seven essential $C$. albicans genes were proposed for the analysis [52]. This set includes $A A T 1 a$, $A C C 1, A D P 1, S Y A 1, V P S 13, Z W F 1 b$, and MPIB, which has since been renamed PMI1 [53]. MLST proved to be a useful method for epidemiological differentiation of clinical isolates of $C$. albicans $[49,51]$.

Tavanti, et al. [54] found MLST on a panel of 416 isolates of $C$. albicans from different sources recognized a population structure comprising four major clades and eight minor clades. Odds, et al. [55] evaluated larger panel of C. albicans isolates (1391) for MLST analysis, the number of clades recognized increased to clades 17. ABC types (based on the presence or absence of an intron in rDNA) and geographical origins showed statistically significant variations among clades, but anatomical source and antifungal-susceptibility data were not significantly associated. Computational haplotype analysis of the gene fragments sequenced for MLST showed a high frequency of recombination events, which suggests that $C$. albicans isolates had mixed evolutionary histories resembling those of a sexually reproducing species [55]. Also mitochondrial genes have been demonstrated by Wang, et al. [56], to be promising targets for genotyping and population genetics of $C$. albicans.

Ge, et al. [57] evaluated C. albicans isolates from the vagina and oral cavity of Chinese candidose vulvovaginal patients and asymptomatic carriers. The genotypes of these strains were identified. Antifungal susceptibility testing revealed that the two dominant genotypes, CAI 30-45 and CAI 32-46 associated with vulvovaginitis showed significantly different azole-susceptibility. Different mutation patterns in the azole target gene ERG11 were correspondingly observed among C. albicans isolates representing different genotypes and sources. Isolates with the same or similar CAI genotypes usually possessed identical or phylogenetically closely related ERG11 sequences. Loss of heterozygosity in ERG11 was observed in all the CAI 32-46 isolates but not in the CAI 30-45 isolates and most of the oral isolates sequenced. Compared with the ERG11 sequence of strain
SC5314, two homozygous nonsynonymous substitutions, leading to two amino-acid changes (A114S and Y257H) in the Erg11p were found in CAI 32-46 isolates. The association between azole-susceptibility and $C$. albicans genotype may be of potential therapeutic important.

Dodgson, et al. [58] developed MLST for C. glabrata through amplification and sequencing of fragments from the coding regions of six genes (FKS, LEU2, NMT1, TRP1, UGP1, and URA3). An MLST analysis of 230 C. glabrata isolates from five populations that differed both geographically and temporally confirmed that using the six loci, it was possible to assess genetic diversity and differentiation among isolates of this species [59].

Tavanti, et al. [60] described a high degree of reproducibility and discriminatory power above $99 \%$ when using MLST to differentiate between isolates of $C$. tropicalis through sequencing of polymorphic fragments of six genes (MDR1, ICL1, SAPT2, SAPT4, $X Y R 1$, and $Z W F 1 a$ ). The method could differentiate between 87 of the 106 DST isolates tested. Jacobsen, et al. [61] later conducted a MLST study of the $C$. tropicalis molecular phylogeny with 242 isolates; the haplotype analysis revealed several recombination events.

While conducting a molecular characterization study of 61 isolates of $C$. tropicalis, Magri, et al. [62] found that only 3 isolates were resistant to fluconazole, but it was not possible to correlate the Diploid Sequence Types (DSTs) with resistance. These same authors reported that MLST is an important tool for the study of genetic diversity, particularly with regard to polymorphisms. Although many molecular studies have been conducted to analyze the different Candida species, more research is needed to investigate the genetic and phylogenetic diversities of Candida species and their relation to epidemiology. The development of next-generation sequencing (NGS) over recent years has allowed a technological breakthrough in the molecular characterization of Candida species. This technology allows accurate and thorough genotyping of genes involved in antifungal resistance in strains clinical. Garnoud, et al. [63] used NGS to investigate echinocandin and azole resistance in clinical Candida isolates. Six genes involved in antifungal resistance (ERG3, ERG11, TAC1, CgPDR1, FKS1 and FKS2) were analyzed in 40 Candida isolates (18 C.albicans, 15 C.glabrata and 7 C.parapsilosis). A total of 391 SNPs were detected, among which 6 coding SNPs were reported for the first time. Novel genetic alterations were detected in both azole and echinocandin resistance genes. A C. glabrata strain, which was resistant to echinocandins but highly susceptible to azoles, harboured an FKS2 S663P mutation plus a novel presumed lossof-function CgPDR1 mutation. Another C. glabrata isolate, carried a new FKS2 S663A mutation and a new putative gain-of-function CgPDR1 mutation (T370I). This study shows that NGS can be used for extensive assessment of genetic mutations involved in antifungal resistance.

Genome sequencing of Candida species provides the opportunity to elucidate some of the mechanisms involved in intrinsic or acquired resistance by yeasts of the Candida genus. The omics technologies (genome, transcriptome, proteome, 
metaboiome, microbiome and mycobiome) will become very valuable for detecting mechanisms of resistance in clinical strains subjected to multidrug pressure. The high-throughput sequencing methods still has little practicability and feasibility in daily clinical practice and thus still remains challenging, but as future perspectives, current rapid progression of automation of these technologies makes their upcoming routine application likely.

\section{Acknowledgements}

This review did part of project supported by a grant from the Foundation to Support the Development of Education, Science and Technology of Mato Grosso do Sul ( FUNDECT ) and the Higher Education Personnel Improvement Coordination (CAPES), Mato Grosso do Sul, Brazil.

\section{References}

1. Yang YL. Virulence factors of Candida species. J Microbiol Immunol Infect. 2003;36(4):223-8.

2. Baixench MT, Aoun N, Desnos-Ollivier M, Garcia-Hermoso D, Bretagne $\mathrm{S}$, et al. Acquired resistance to echinocandins in Candida albicans: case report and review. J. Antimicrob. Chemother. 2007;59(6):1076-83.

3. Barker SKP, Rogers DP. Recent insights into the mechanisms of antifungal resistance. Curr Infect DisRep. 2006;8(6):449-56.

4. Barbedo LS, Sgarbi DBG. Candidíase. J. Bras. Doenças Sex. Transm. 2010;22(1):22-38.

5. Giolo MP, Svidzinski TIE. Fisiopatogenia, epidemiologia e diagnóstico laboratorial da candidemia. J. Bras. Patol. Med. Lab. 2010;46(3):225234.

6. Butler G. Fungal Sex and Pathogenesis. Clin. Microbiol. Rev. 2010;23(1):140-159.

7. Colombo AL, Guimarães T. Epidemiologia das infecções hematogênicas por Candida spp.Rev. Soc. Bras. Med. Trop.2003;36(5):599-607.

8. Gómez J, García-Vázquez E, Hernández A, Espinosa C, Ruiz J. Candidemiasnosocomiales: nuevos retos de um problema emergente. Rev. Esp. Quimiot. 2010;23(4):158-168.

9. Nguyen K, Zmeter G, Claris O, Kassai B. Epidemiology of invasive Candida infection in a neonatal intensive care unit in France. ActaPediat. 2012;101:137-139.

10. Pfaller MA, Diekema DJ. Epidemiology of Invasive Candidiasis: a Persistent Public Health Problem. Clin. Microbiol. Rev.2007;20(1):133163.

11. Nucci M and Colombo AL. Candidemia due to Candida tropicalis: clinical, epidemiologic, and microbiologic characteristics of 188 episodes occurring in tertiary care hospitals. DiagnMicrobiol Infect Dis. 2007;58(77):82.

12. Sipsas NV, Lewis RE, Tarrand J, Hachem R, Rolston KV, Raad II et al. Candidemia in patients with hematologic malignancies in the era of new antifungal agents (2001-2007): stable incidence but changing epidemiology of a still frequently lethal infection.Cancer. 2009;115:4745-4752.

13. Godoy P, Tiraboschi IN, Severo LC, Bustamante B, Calvo B, De Almeida LP, et al. Species Distribution and Antifungal Susceptibility Profile of Candida spp. Bloodstream Isolates from Latin American Hospitals. Mem. Inst. Oswaldo Cruz. 2003;98(3):401-405.
14. Almeida AA, Mesquita CSS, Svidzinski TIE, Oliveira KMP. Antifungal susceptibility and distribution of Candida spp. isolates in the University Hospital in the municipality of Dourados, Mato Grosso do Sul State, Brazil. Rev. Soc. Bra. Med. Trop. 2013;46:335-339.

15. Goldman M, Pottage Júnior JC, Weaver DC. Candida kruseifungemia. Report of 4 cases and review of the literature. Medicine [Baltimore]. 1993;72(3):143-150.

16. TrofaD, Gácser A, Nosanchuk JD. Candida parapsilosis, an emerging fungal pathogen. Clin. Microbiol. Ver. 2008; 21(4):606-625.

17. Brito LR, Guimaraes T, Nucci M, Rosas RC, Paula Almeida L, Da Matta DA, et al. Clinical and microbiological aspects of candidemia due to Candida parapsilosis in Brazilian tertiary care hospitals.Med. Mycol. 2006;44(3): 261-266.

18. Asbeck EC, Huang Y, Markham AN, Clemons KV, Stevens DA. Candida parapsilosisfungemia in neonates: genotyping results suggest healthcare workers hands as source, and review of published studies. Mycopathologia. 2007;164:287-293.

19. Spolidorio DMP, Boriollo MFG, Carlos Estrela C, Spolidorio LC. Diferentes métodos fenotípicos para isolamento e identificação de espécies deCandida. Robrac. 2009;18(45):18-26.

20. Higashi CM, Takashina FH, Rechenchoski ZD3, Stipp-Abe AT, Vespero EC, Quesada RMB, et al. Comparação do sistema de identificação automatizado Vitek 2 e PCR-ITS para caracterização das espécies dos isolados clínicos de Candidaspp. Ciências Biológicas e da Saúde. 2015;36(1):233-242.

21. Meletiadis J, Arabatzis M, Bompola M, Tsiveriotis K, Hini S, Petinaki $\mathrm{E}$, et al. Comparative evaluation of three commercial identification systems using common and rare bloodstream yeast isolates. J. Clin. Microbiol. 2011; 49(7):2722-2727.

22. Kumar S, Vyas A, Kumar M, MehraSK. Application ofCHROMagarCandida for identification of clinically important Candida species and their antifungal susceptibility pattern. IJBMR. 2013;4(4):3600-3606.

23. Braun BR, Van Het Hoog MVH, D'enfert C, Martchenko M, Dungan J, Kuo A, et al. A human-curated annotation of the Candida albicans genome. Plos Genet 2005;1(1):36-57.

24. Jones T, Federspiel NA, ChibanaH, Dungan J, Kalman S, Magee BB, et al. The diploid genome sequence of Candida albicans. PNAS. 2004;101:7329-7334.

25. Van Het Hoog, MVH, Rast TJ, Martchenko M, Grindle S, Dignard $\mathrm{D}$, Hogues $\mathrm{H}$, et al. Assembly of the Candida albicans genome into sixteen supercontigs aligned on the eight chromosomes. Genome Biol. 2007;8(4):article R52.

26. Butler G, Rasmussen MD, Lin MF, Santos MA, Akthikumar S, Munro $\mathrm{CA}$, et al. Evolution of pathogenicity and sexual reproduction in eight Candida genomes. Nature. 2009;459:657-662.

27. Dujon B, Sherman D, Fischer G, Durrens P, Casaregola I, Lafontaine I, et al. Genome evolution in yeast. Nature. 2004;4303:5-44.

28. Bridge PD, Spooner BM, Roberts PJ. The impact of molecular data in fungal systematic. Adv. Botanical Res. 2005;42:34-68.

29. Hebert PD, Cywinska A, Ball SL, Dewaard JR. Biological identifications through DNA barcodes. Proc. R. Soc. Lond. B2003;270(1512):313-21.

30. Schindel, DE, Miller, SE. DNA barcoding a useful tool for taxonomists. Nature. 2005;435(7038):17.

31. Seifert KA, Samson RA, Dewaard JR, Houbraken J, Lévesque CA, Moncalvo JM, et al. Prospects for fungus identification using CO1 DNA barcodes, with Penicillium as a test case. PNAS. 2007;104:390-3906. 
32. Dentinger BTM, Didukh MY, Moncalvo JM. Comparing COI and ITS as DNA barcode markers for mushrooms and allies (Agaricomycotina). PLoS One. 2011;6:1-8.

33. Simon L, BousquetJ, Levesque RC, Lalonde M. Origin and diversification of endomycorrhizal fungi and coincidence with vascular land plants. Nature. 1993;363:67-69.

34. Shokohi T, SotehMbh, PouriZs, Hedayati Mt, Mayahi S. Identification of Candida species using PCR-RFLP in cancer patients in Iran. Indian J. Med. Microbiol. 2010;28(2):147-151.

35. Schoch CL, Seifert KA, Huhndorf S, Robert V, Spouge JL, Levesque CA, et al. Nuclear ribosomal internal transcribed spacer (ITS) region as a universal DNA barcode marker for Fungi. PNAS. 2012;109(16):62416.

36. Kurztzman, CP, Albertyn J, Basehoar-Powers E. Multigene phylogenetic analysis of the Lipomycetaceae and the proposed transfer of Zygozyma species to Lipomyces and Babjeviaanomala to Dipodascopsis. FEMS Yast Research. 2007;7(6):1027-34

37. Brinkman FS, Leipe DD. Phylogenetic analysis. Methods Biochem. Anal. 2001;43:323-58.

38. Russo CAM, Miyaki CY, Pereira SL. Reconstrução filogenética: Métodos geométricos. In Matioli SR, Fernandes FMC, editors. Biologia Molecular e Evolução. Brasil, Holos. 2012; p.123-132.

39. Pereira SL, Miyaki CY, Russo CAM. Reconstrução filogenética: Métodos probabilísticos. In Matioli SR, Fernandes FMC, editors. Biologia Molecular e Evolução. Brasil, Holos. 2012;p.133-146.

40. Huelsenbeck JP, Ronquist F. Bayseane analysis of molecular evolution using MrBayes. In Nielsen R, editor. Statistical Methods In Molecular Evolution. Springer; 2005; p.183-232.

41. Cardona-Castro N, Revankar SG, Ortiz P, Cuervo C, Kirkpatrick WR, Mcatee RK, et al. Proteinase detection, DNA typing and antimycotic susceptibility of Candida isolates from Colombian women with vulvovaginal candidiasis. Rev. Iberoam. Micol. 2002;19(2): 89-94.

42. Pujol C, Pfaller M, Soll DR. CA3 fingerprinting of C. albicans bloodstream isolates from the United States, Canada, South America and Europe reveals a European clade. J. Clin. Microbio. 2002;40(8):2729-2740.

43. Rho J, Shin JH, Song JW, Park MR, Kee SJ, Jang SJ, et al. Molecular investigation of two consecutive nosocomial clusters of Candida tropicaliscandiduria using pulsed-field gel electrophoresis. J. Microbiol. 2004;2(42):80-86.

44. Pinto PM, Resende MA, Koga-Ito CY, Tendler M. Genetic variability analysis among clinical Candida spp. isolates using random amplified polymorphic DNA.Mem. Inst. Oswaldo Cruz. 2004;99(2):147-152.

45. Boriollo MF, Rosa EA, Gonçalves RB, Höfling JF. Parity among interpretation methods of MLEE patterns and disparity among clustering methods in epidemiological typing of Candida albicans. J. Microbiol. Methods. 2006;64(3):346-65.

46. Sampaio P, Nogueira E, Loureiro AS, Delgado-Silva Y, Correia A, Pais C. Increased number of glutamine repeats in the C-terminal of Candida albicans Rlm1p enhances the resistance to stress agents. Antonie Van Leeuwenhoek. 2009;96(4):395-404.

47. Cummings CA, Relman DA. Using DNA microarrays to study hostmicrobe interactions. Emerg Infect Dis. 2000; 6:513-25.

48. Maiden MC, Bygraves JA, Feil E, Morelli G, Russell JE, Urwin R, et al. Multilocus sequence typing: a portable approach to the identification of clones within populations of pathogenic microorganisms. PNAS. 1998;95:3140-3145.

49. BougnouxME, Morand S, D'enfert C. Usefulness of multilocus sequence typing for characterization of clinical isolates of Candida albicans. J. Clin. Microbiol. 2002;40(4):1290-1297.

50. Odds FC, Jacobsen MD. Multilocus sequence typing of pathogenic of Candida species. Eukaryot. Cell. 2008; 7:1075-1084.

51. Tavanti A, Gow NAR, Senesi S, Maiden MCJ, Odds FC. Optimization and validation of multilocus sequence typing for Candida albicans. J. Clin. Microbiol. 2003;8:3765-3776.

52. Bougnoux ME, Tavanti A, Bouchier C, GowNA, Magnier A, Davidson $\mathrm{AD}$, et al. Collaborative consensus for optimized multilocus sequence typing of Candida albicans. J. Clin. Microbiol 2003;41(11):5265-5266.

53. Arnaud MB, Costanzo MC, Skrzypek MS, Shah P, Binkley G, Lane C, et al. Sequence resources at the Candida genome database. Nucleic Acids Res. 2007;35(1):D452-D456.

54. Tavanti A, Davidson AD, Fordyce MJ, Gow NA, Maiden MCJ, Odds FC. Population structureand properties of Candidaalbicans, as determined by multilocus sequence typing. J Clin Microbiol. 2005;43:5601-5613.

55. Odds FC, Bougnoux ME, Shaw DJ, Bain JM, Davison AD, Diogo D, et al. Molecular phylogenetics of Candida albicans. Eukaryot Cell. 2007;6:1041-1052.

56. Wang H, Guo H, Sun S, Xu J. Abundant sequence variation around the mitochondrial origin of replication in the human opportunistic yeast pathogen Candida albicans from a tropical island in China. Fungal Genet Biol. 2007;44:991-1001.

57. Ge SH, Wan Z, Li J, Xu J, Li RY, Bai FY. Correlation between azole susceptibilities, genotypes, and ERG11 mutations in Candida albicans isolates associated with vulvovaginal candidiasis in China. Antimicrob Agents Chemother. 2010;54:3126-3131.

58. Dodgson AR, Pujol C, Denning DW, Soll DR, Fox AJ. Multilocus sequence typing of Candida glabrata reveals geographically enriched clades. J. Clin. Microbiol. 2003;41(12):5709-5717.

59. Lott TJ, Frade JP, Lockhart SR. Multilocus sequence type analysis reveals both clonality and recombination in populations of Candida glabrata bloodstream isolates from U.S. surveillance studies. Eukaryot Cell. 2010;9(4): 619-625.

60. Tavanti A, Davidson AD, Johnson EM, Maiden MC, Shaw DJ, Gow NA, et al. Multilocus sequence typing for differentiation of strains Candida tropicalis. J. Clin. Microbiol. 2005;43:5593-5600.

61. Jacobsen MD, Rattray AM, Gow NA, Odds FC, Shaw DJ. Mitochondrial haplotypes and recombination in Candida albicans. Med. Mycol. 2008;46:647-654.

62. Magri MMC, Gomes-Gouvêa MS, Freitas VLT, Motta AL, Moretti ML, Shikanai-Yasuda MA. Multilocus sequence typing of Candida tropicalisshows the presence of differentclonal clusters and fluconazole susceptibility profiles in sequential isolates from candidemia patients in São Paulo, Brazil. J. Clin. Microbiol. 2013;51(1):268-277.

63. Garnttaud C, Boerel F, Sertour N, Bougnoux ME, Dannaoui E, Larrat $S$, et al. Next-generation sequencing offers new insights into the resistance of Candida spp. to echinocandins and azoles. J. Antimicrob. Chemother. 2015;70(9):2556-65. 